\title{
THE INFLUENCE OF SOLVENT QUALITY ON THE STATIC PROPERTIES OF A LINEAR POLYMER: A MOLECULAR DYNAMICS STUDY
}

\author{
B. SMIT ${ }^{1}$, A. VAN DER PUT, C.J. PETERS, J. DE SWAAN ARONS \\ Laboratorium voor Anorganische en Fysische Chemie, Technische Universiteit Delft, \\ P.O. Box 5045, 2600 GA Delft, The Netherlands
}

and

J.P.J. MICHELS

Van der Waals Laboratorium, Universiteit van Amsterdam, P.O. Box 20216, 1000 HE Amsterdam, The Netherlands

Received 24 October 1987; in final form 20 January 1988

\begin{abstract}
We present molecular dynamics simulations of a polymer in a solvent of monomers. We have studied the influence of the "quality" of the solvent on the radius of gyration. This is modelled by varying the $\epsilon_{\mathrm{mp}}$ of a Lennard-Jones potential, which describes the interaction of the monomers with the polymer segments. Starting from an isolated chain $\left(\epsilon_{\mathrm{mp}}=0\right)$, the introduction of a very small interaction with the solvent causes a large decrease in the radius of gyration. A further increase of $\epsilon_{\mathrm{mp}}$ causes the radius of gyration to increase again.
\end{abstract}

\section{Introduction}

Computer simulations of polymer systems, utilizing Monte Carlo or molecular dynamics methods, can provide detailed quantitative data on precisely defined models. However, most previous studies on polymer systems have dealt with isolated chains. The influence of the solvent has been represented qualitatively by the temperature, calculated from the Gibbs distribution of the chain [1] or approximated by a random force on the polymer segments, using the Brownian dynamics technique [2].

Accounting for the solvent particles gives rise to a large increase in the amount of computer time, not only because of the increase in the total number of particles but also because of the longer relaxation time of the polymer due to the solvent. Therefore it is important to study to what extent the solvent influences the properties of a polymer.

\footnotetext{
1 Present address: Koninklijke/Shell Laboratorium Amsterdam (Shell Research B.V.), P.O. Box 3003, 1003 AA Amsterdam, The Netherlands.
}

In several molecular dynamics studies the influence of the solvent on the properties of linear polymers has been studied. Bruns and Bansal [3,4] found that the radius of gyration was slightly higher for an isolated chain than for a chain immersed in a solvent. Comparison of the data from Bishop et al. [5] and Rapaport [6] for an isolated chain with the data for a chain in a solvent [7-9] showed almost no solvent effect. This controversy motivated Khalatur et al. [10] to study in detail the influence of the solvent on the static properties of a polymer. In this study Khalatur et al. concluded that the static structure of a polymer is mainly determined by intrachain rather than by solvent effects, i.e. the solvent had almost no effect on the static properties. In contrast to this conclusion, we demonstrated [11] that the static properties of a star polymer are significantly influenced by the density of the solvent.

Recently Toxvaerd [12] studied the effect of the solvent on the structure and dynamics of a polymer by solving the generalized Langevin equation. In this work Toxvaerd showed that a free polymer has a 
slightly higher radius of gyration than a polymer in a solvent.

It is well known that the shape of a polymer is influenced by the chemical nature of the solvent. However, all previous studies concerned a polymer immersed in a solvent containing its own segments (i.e. the same Lennard-Jones potential is operating between all particles). It is therefore interesting to study the influence of the quality (chemical nature) of the solvent, which is modelled by varying only the interactions between the polymer segments and the monomers of the solvent.

Although we have tried to incorporate some of the characteristics of real polymer systems, our model and the introduced concept of the solvent quality are, of course, far from accurate descriptions of real polymers.

\section{Model and computational details}

In this study we have used a linear polymer having 8 segments in a solvent containing 492 monomers. The interaction potential consists of two parts: a shifted Lennard-Jones potential which operates between all 500 particles and a harmonic potential which links the 8 particles into a linear polymer. The harmonic potential is given by

$U_{i j}^{\mathrm{H}}=\frac{1}{2} k\left(\left|\boldsymbol{r}_{i}-\boldsymbol{r}_{j}\right|-\sigma\right)^{2}$

for $i=1,2, \ldots, 7$ and $j=i+1$, where the value of the force constant is made sufficiently large such that $98 \%$ of the connected units have an instantaneous length within $2 \%$ of the average value. The shifted LennardJones potential is given by

$$
\begin{aligned}
U^{\mathrm{LJ}}\left(r_{i j}\right)= & 4 \epsilon_{i j}\left[\left(\sigma / r_{i j}\right)^{12}-\left(\sigma / r_{i j}\right)^{6}\right]+U\left(r_{\mathrm{c}}\right) \\
& r_{i j} \leqslant 2.5 \sigma, \\
=0 \quad & r_{i j}>2.5 \sigma,
\end{aligned}
$$

with $r_{\mathrm{c}}=2.5 \sigma$.

Usually it is assumed that the polymer is immersed in a solvent of its own segments, this implies

$$
\begin{aligned}
& \epsilon_{\mathrm{mm}}=\epsilon_{\mathrm{pp}}=\epsilon^{0}, \\
& \epsilon_{\mathrm{mp}}=\sqrt{\epsilon_{\mathrm{mm}} \epsilon_{\mathrm{pp}}}=\epsilon^{0}, \\
& \sigma_{\mathrm{mp}}=\sigma_{\mathrm{mm}}=\sigma_{\mathrm{pp}}=\sigma .
\end{aligned}
$$

The subscript mm indicates monomer-monomer interactions, pp polymer segment-polymer segment interactions and mp monomer-polymer segment interactions.

One method of changing the quality of the solvent is to vary the polymer-monomer interactions by introducing the parameter $\lambda$ :

$$
\epsilon_{\mathrm{mp}}=\lambda \sqrt{\epsilon_{\mathrm{mm}} \epsilon_{\mathrm{pp}}}=\lambda \epsilon^{0} \text {. }
$$

When $\lambda<1$ the quality of the solvent is poorer than the reference $\left(\epsilon^{0}\right)$ and when $\lambda>1$ the quality of the solvent is better than the reference.

Throughout this paper the usual reduced units are adopted. Periodic boundary conditions are imposed in all three directions. The equations of motion are solved using Verlet's [13] integration scheme. All the simulations have been performed at a reduced temperature $T^{*}=2.0$ and a reduced density $\rho^{*}=0.6$.

\section{Results}

The radius of gyration $\langle S\rangle$ is widely used to indicate the size of a chain and is defined by

$S^{2}=\frac{1}{N_{\mathrm{p}}} \sum_{j=1}^{N_{\mathrm{p}}}\left|\boldsymbol{r}_{j}(t)-Z(t)\right|^{2}$,

with $N_{\mathrm{p}}$ the number of polymer segments $\left(N_{\mathrm{p}}=8\right)$, $r_{j}(t)$ the position of the $j$ th polymer segment at time step $t, Z(t)$ the centre of mass of the polymer.

In order to avoid large correlations, due to the long relaxation time of the polymer, we have skipped a number of time steps between two samples of the radius of gyration. This number of time steps is obtained by monitoring the autocorrelation function of the radius of gyration. Estimates of the standard deviations are obtained by dividing the samples into five groups and calculating the group averages.

The values of the radius of gyration for several values of $\lambda$ are presented in table 1 and fig. 1 . These data show a significant influence of the quality of the solvent on the radius of gyration of the polymer. In a forthcoming paper we will extend this study to other models and we will also present time-dependent properties of the polymer.

Fig. 1 demonstrates that, compared to an isolated chain $(\lambda=0)$, the introduction of a very small interaction of the polymer segments with the mono- 
Table 1

Influence of the quality of the solvent on the radius of gyration of a polymer. The reduced temperature for each simulation was $T^{*}=2.0$ and the reduced density was $\rho^{*}=0.6$

\begin{tabular}{lll}
\hline No. & $\lambda$ & $\left\langle S^{2}\right\rangle$ \\
\hline 1 & 0.00 & $2.13 \pm 0.02$ \\
2 & 0.0002 & $1.75 \pm 0.05$ \\
3 & 0.02 & $1.67 \pm 0.06$ \\
4 & 0.20 & $1.59 \pm 0.07$ \\
5 & 0.60 & $1.66 \pm 0.09$ \\
6 & 1.00 & $1.91 \pm 0.06$ \\
7 & 1.40 & $2.15 \pm 0.05$ \\
\hline
\end{tabular}

mers of the solvent gives rise to a decrease of the radius of gyration of $25 \%$. As the interaction is further increased the radius of gyration starts to increase again.

Note that if attention is restricted only to an isolated chain $(\lambda=0)$ and a chain immersed in its seg-

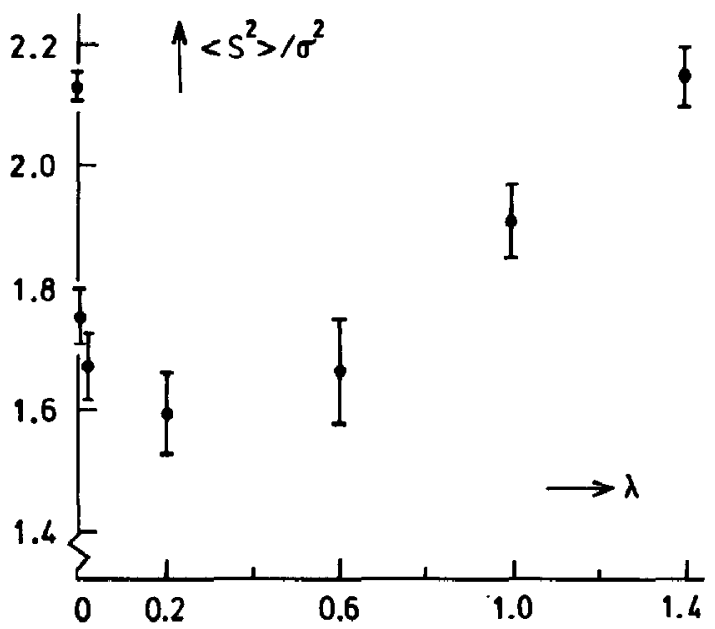

Fig. 1. The mean-square radius of gyration as a function of the quality of the solvent. ments $(\lambda=1)$ the results suggest that the influence of the solvent is not very pronounced. From this point of view it can be understood that depending on the details of the simulation, some molecular dynamics simulations (viz. refs. [3,4,7-10]) show almost no solvent effect, but it refutes the conclusion that the static properties of a polymer are mainly determined by intrachain interactions rather than by solvent effects.

\section{Acknowledgement}

We have enjoyed stimulating discussions with $M$. Bishop, K.R. Cox, D. Frenkel and M.A.J. Michels.

\section{References}

[1] I. Webman, J.L. Lebowitz and M.H. Kalos, Macromolecules 14 (1981) 1495 .

[2] M. Bishop and J.P.J. Michels, J. Chem. Phys. 84 (1986) 447.

[3] W. Bruns and R. Bansal, J. Chem. Phys. 74 (1981) 2064.

[4] W. Bruns and R. Bansal, J. Chem. Phys. 75 (1981) 5149.

[5] M. Bishop, M.H. Kalos and H.L. Frisch, J. Chem. Phys. 79 (1983) 3500.

[6] D.C. Rapaport, J. Phys. A11 (1978) L213.

[7] M. Bishop, M.H. Kalos and H.L. Frisch, J. Chem. Phys. 70 (1979) 1299.

[8] H.L. Frisch, M. Bishop, D. Ceperley and M.H. Kalos, J. Macromol. Sci. Phys. B18 (1980) 453.

[9] D.C. Rapaport, J. Chem. Phys. 71 (1979) 3299.

[10] P.G. Khalatur, Yu.G. Papulov and A.S. Pavlov, Mol. Phys. 58 (1986) 887.

[11] B. Smit, A. van der Put, C.J. Peters, J. de Swaan Arons and J.P.J. Michels, J. Chem. Phys., to be published.

[12] S. Toxvaerd, J. Chem. Phys. 86 (1987) 3667.

[13] L. Verlet, Phys. Rev. 159 (1967) 98. 